\title{
Linear independence of powers of singular moduli of degree 3
}

\author{
Florian Luca, Antonin Riffaut
}

August 9, 2018

\begin{abstract}
We show that two distinct singular moduli $j(\tau), j\left(\tau^{\prime}\right)$, such that for some positive integers $m, n$ the numbers $1, j(\tau)^{m}$ and $j\left(\tau^{\prime}\right)^{n}$ are linearly dependent over $\mathbb{Q}$ generate the same number field of degree at most 2 . This completes a result of Riffaut, who proved the above theorem except for two explicit pair of exceptions consisting of numbers of degree 3 . The purpose of this article is to treat these two remaining cases.
\end{abstract}

\section{Introduction}

Let $j$ be the classical $j$-function on the Poincare plane $\mathbb{H}=\{z \in \mathbb{C}: \operatorname{Im} z>0\}$. A singular modulus is a number of the form $j(\tau)$, where $\tau \in \mathbb{H}$ is a complex algebraic number of degree 2. It is known that $j(\tau)$ is an algebraic integer and Class Field Theory tells that

$$
[\mathbb{Q}(j(\tau)): \mathbb{Q}]=[\mathbb{Q}(\tau, j(\tau)): \mathbb{Q}(\tau)]=h_{\Delta}
$$

is the class number of the order $\mathcal{O}_{\Delta}=\mathbb{Z}[(\Delta+\sqrt{\Delta}) / 2]$, where $\Delta$ is the discriminant of the minimal polynomial of $\tau$ over $\mathbb{Z}$. Moreover, $\mathbb{Q}(\tau, j(\tau)) / \mathbb{Q}(\tau)$ is an abelian Galois extension with Galois group (canonically) isomorphic to the class group of the order $\mathcal{O}_{\Delta}$. One can also interpret $\mathcal{O}_{\Delta}$ as the automorphism ring of the lattice $\langle 1, \tau\rangle$, or of the corresponding elliptic curve. For all details, see, for instance, [7, §7 and §11].

Starting from the ground-breaking article of André [2], equations involving singular moduli were studied by many authors, see [1, 5, 10, for a historical account and further references. In particular, Kühne [8] proved that the equation $x+y=1$ has no solutions in singular moduli $x, y$, and Bilu et al. [4 proved the same conclusion holds for the equation $x y=1$. These results were generalized in [1] and [5]. In [1, solutions of all linear equations $A x+B y=C$, with $A, B, C \in \mathbb{Q}$, were determined. Here is the main result of [1].

Theorem 1.1 (Allombert et al. [1]). Let $x, y$ be two singular moduli, and $A, B, C$ rational numbers with $A B \neq 0$. Assume that $A x+B y=C$. Then we have one of the following options:

(trivial case) $A+B=C=0$ and $x=y$;

(rational case) $x, y \in \mathbb{Q}$; 
(quadratic case) $x \neq y$ and $x, y$ generate the same number field over $\mathbb{Q}$ of degree 2.

This result is best possible, since in both the rational case and the quadratic case of Theorem 1.1, one easily finds $A, B, C \in \mathbb{Q}$ such that $A B \neq 0$ and $A x+B y=C$. Moreover, the lists of singular moduli of degrees 1 and 2 over $\mathbb{Q}$ are widely available or can be easily generated using a suitable computer package, like PARI 11. In particular, there are 13 rational singular moduli, and 29 pairs of $\mathbb{Q}$-conjugate singular moduli of degree 2 ; see [5, Section 1] for more details. This means that Theorem 1.1 gives a completely explicit characterization of all solutions.

In 10, Riffaut generalized Theorem 1.1 by introducing exponents; that is, instead of equation $A x+B y=C$, he considered the more general equation $A x^{m}+B y^{n}=C$, where the positive integer exponents $m, n$ are unknown as well. He proved that, if $x \neq y$, then $x, y$ generate the same number field of degree $h \leq 3$, and $h=3$ is possible only if either $\left\{\Delta, \Delta^{\prime}\right\}=\{-4 \cdot 23,-23\}$, or $\left\{\Delta, \Delta^{\prime}\right\}=\{-4 \cdot 31,-31\}$, where $\Delta, \Delta^{\prime}$ denote the respective discriminants of $x$ and $y$. In this article, we eliminate these two remaining cases. Here is the statement of our result.

Theorem 1.2. Let $x=j(\tau), y=j\left(\tau^{\prime}\right)$ be two singular moduli of respective discriminants $\Delta$ and $\Delta^{\prime}$, and $m, n$ two positive integers. If $\left\{\Delta, \Delta^{\prime}\right\}=$ $\{-4 \cdot 23,-23\}$ or $\left\{\Delta, \Delta^{\prime}\right\}=\{-4 \cdot 31,-31\}$, then the numbers $1, x^{m}, y^{n}$ are linearly independent over $\mathbb{Q}$.

Consequently, Theorem 1.2 together with [10, Theorem 1.5] completely solve the above equation for distinct singular moduli, and we deduce the following Theorem.

Theorem 1.3. Let $x=j(\tau), y=j\left(\tau^{\prime}\right)$ be two distinct singular moduli of respective discriminants $\Delta$ and $\Delta^{\prime}$, and $m, n$ two positive integers. Assume that $A x^{m}+B y^{n}=C$, for some $A, B, C \in \mathbb{Q}^{\times}$. Then $x$ and $y$ generate the same number field over $\mathbb{Q}$ of degree at most 2 .

As previously, this result is now best possible for distinct singular moduli, since if $h \leq 2$, then for all exponents $m, n$, one easily finds $A, B, C \in \mathbb{Q}^{\times}$such that $A x^{m}+B y^{n}=C$. However, our current methods are still not able to handle the case $x=y$, which is equivalent to the following question: can a singular modulus of degree 3 or higher be a root of a trinomial with rational coefficients? Much about trinomials is known, but this knowledge is still insufficient to rule out such a possibility. Otherwise, the assumption $C \neq 0$ is seemingly restrictive, but in fact, the case $C=0$ is contained in [10, Theorem 1.6].

Our calculations were performed using the PARI/GP package [11]. The sources are available from the second author.

\section{Preliminaries}

Below we briefly recall some basic facts about the conjugates of a singular modulus and the height of an algebraic number. 
Fields generated by a power of a singular modulus

Let $j(\tau)$ be a singular modulus of discriminant $\Delta$. It is well-known that the conjugates of $j(\tau)$ over $\mathbb{Q}$ can be described explicitly; see, for instance, [10, Subsection 2.2]. In particular, $j(\tau)$ admits one real conjugate which has the property that it is much larger in absolute value than all its other conjugates, called the dominant $j$-value of discriminant $\Delta$. As a useful consequence, a singular modulus and any of its powers generate the same field over $\mathbb{Q}$; see $[10$, Lemma 2.6], a statement which we reproduce below.

Lemma 2.1. Let $x$ be a singular modulus of discriminant $\Delta$, with $|\Delta| \geq 11$, and $n$ a non-zero integer. Then $\mathbb{Q}(x)=\mathbb{Q}\left(x^{n}\right)$.

The height of a non-zero algebraic number

Let $\alpha$ be a non-zero algebraic number of degree $d$ over $\mathbb{Q}$, and $\alpha_{1}=$ $\alpha, \alpha_{2}, \ldots, \alpha_{d}$ all its conjugates in $\overline{\mathbb{Q}}$. The logarithmic height of $\alpha$, denoted by $\mathrm{h}(\alpha)$, is defined to be

$$
\mathrm{h}(\alpha)=\frac{1}{d}\left(\log |a|+\sum_{k=1}^{d} \log \max \left\{1,\left|\alpha_{k}\right|\right\}\right),
$$

where $a$ is the leading coefficient of the minimal polynomial of $\alpha$ in $\mathbb{Z}$. In particular, $\log |a|=0$ when $\alpha$ is an algebraic integer.

Here are some useful properties of the logarithmic height.

- For any non-zero algebraic number $\alpha$ and $\lambda \in \mathbb{Q}^{*}$, we have $\mathrm{h}\left(\alpha^{\lambda}\right)=$ $|\lambda| \mathrm{h}(\alpha)$. In particular, $\mathrm{h}(1 / \alpha)=\mathrm{h}(\alpha)$. See [6, Lemma 1.5.18].

- For any two non-zero algebraic numbers $\alpha$ and $\beta$, we have $\mathrm{h}(\alpha \beta) \leq \mathrm{h}(\alpha)+$ $\mathrm{h}(\beta)$.

\section{Linear forms in two logarithms}

Let $\alpha$ be an algebraic number with $|\alpha|=1$ but not a root of unity and $m$ a positive integer. We are interested in estimating the quantity $\lambda=1-\alpha^{n}$, which is closely related to a linear form in two logarithms.

Laurent, Mignotte and Nesterenko describe in [9] a lower bound on the absolute value of a general linear form in two logarithms, see [9, Théorème 3]. In our particular case, Mignotte give in [3] a slight sharpening of this bound. The following Theorem is a corollary of [3, Theorems A.1.2 and A.1.3].

Theorem 3.1. Let $\alpha$ be a complex algebraic number with $|\alpha|=1$, but not a root of unity, and $m$ a positive integer. There exists an effective computable constant $c_{1}(\alpha)>0$, depending only on the degree $d$ of $\alpha$ over $\mathbb{Q}$ and its logarithmic height $\mathrm{h}(\alpha)$, such that

$$
\left|1-\alpha^{m}\right|>0.99 \mathrm{e}^{-c_{1}(\alpha)(\log m)^{2}} .
$$

Proof. We briefly detail the proof, especially to explain how to compute $c_{1}(\alpha)$ in terms of $d$ and $\mathrm{h}(\alpha)$.

We apply [3, Theorems A.1.2 and A.1.3] to the linear form

$$
\Lambda=2 i \pi-m \log \alpha,
$$


where we choose the principal complex logarithm (defined on $\mathbb{C} \backslash \mathbb{R}^{-}$) for $\log \alpha$. We have

$$
\log |\Lambda|>-\left(9.03 \mathcal{H}^{2}+0.23\right)(D \mathrm{~h}(\alpha)+25.84)-2 \mathcal{H}-2 \log \mathcal{H}-0.7 D+2.07,
$$

where $D=d / 2$ and $\mathcal{H}=D(\log m-0.96)+4.49 \leq c_{1}^{\prime}(d) \log m$ for $m \geq 13$, with

$$
c_{1}^{\prime}(d)=D+\max \left\{0, \frac{4.49-0.96 D}{\log 13}\right\}>0 .
$$

Hence,

$$
\begin{aligned}
& \log |\Lambda|>-(\log m)^{2}\left(9.03 c_{1}^{\prime}(d)^{2}(D \mathrm{~h}(\alpha)+25.84)+\frac{2 c_{1}^{\prime}(d)}{\log m}+\frac{2 \log \log m}{(\log m)^{2}}\right. \\
& \left.+\frac{0.23(D \mathrm{~h}(\alpha)+25.84)+2 \log c_{1}^{\prime}(d)+0.7 D-2.07}{(\log m)^{2}}\right)>-c_{1}(\alpha)(\log m)^{2}
\end{aligned}
$$

with

$$
\begin{aligned}
c_{1}(\alpha)= & 9.03 c_{1}^{\prime}(d)^{2}(D \mathrm{~h}(\alpha)+25.84)+\frac{2 c_{1}^{\prime}(d)}{\log 13}+\frac{2 \log \log 13}{(\log 13)^{2}} \\
& +\frac{0.23(D \mathrm{~h}(\alpha)+25.84)+2 \log c_{1}^{\prime}(d)+0.7 D-2.07}{(\log 13)^{2}} .
\end{aligned}
$$

It follows that

$$
\left|1-\alpha^{m}\right|>\frac{\mathrm{e}^{-c_{1}(\alpha)(\log m)^{2}}}{1+\mathrm{e}^{-c_{1}(\alpha)(\log m)^{2}}}>0.99 \mathrm{e}^{-c_{1}(\alpha)(\log m)^{2}},
$$

resulting from the mean value theorem.

In practice, if $\alpha$ is explicitly known (as an algebraic number in number field $L)$, it is then possible to compute effectively $c_{1}(\alpha)$ for $m \geq 13$. For $m<13$, one just has to estimate directly $\left|1-\alpha^{m}\right|$.

Another way of estimating $1-\alpha^{m}$ is to reduce it modulo a prime ideal $\mathfrak{p}$ of $\mathcal{O}_{L}$. More precisely, we want to evaluate its valuation $v_{\mathfrak{p}}\left(1-\alpha^{m}\right)$ at $\mathfrak{p}$; for an element $z \in L$, we write $v_{\mathfrak{p}}(z)$ instead of $v_{\mathfrak{p}}\left(z \mathcal{O}_{L}\right)$ for more simplicity. This can be obtained as follows.

Proposition 3.2. Let $\alpha$ be an algebraic integer that is not a root of unity in a number field $L$ of degree $d$, and $m$ a positive integer. Let $\mathfrak{p}$ be a prime ideal of $\mathcal{O}_{L}$ over a prime number $p$. Assume that $\mathfrak{p} \nmid \alpha$. Denote by $m_{0}$ the order of $\alpha$ in $\mathcal{O}_{L} / \mathfrak{p}$, that is the least positive integer such that $1-\alpha^{m_{0}}=0 \bmod \mathfrak{p}$, and $v_{0}=v_{\mathfrak{p}}\left(1-\alpha^{m_{0}}\right)$. Then, assuming $p>d+1$, we have

$$
v_{\mathfrak{p}}\left(1-\alpha^{m}\right)= \begin{cases}0 & \text { if } m_{0} \nmid m \\ s v_{\mathfrak{p}}(p)+v_{0} & \text { if } m=m_{0} p^{s} r, \operatorname{gcd}(p, r)=1 .\end{cases}
$$

Proof. If $m_{0} \nmid m$, it is clear that $1-\alpha^{m} \not \equiv 0 \bmod \mathfrak{p}$; hence, $v_{\mathfrak{p}}\left(1-\alpha^{m}\right)=0$. Otherwise, write $m=m_{0} p^{s} r$ with $\operatorname{gcd}(p, r)=1$. We proceed by induction on $s \geq 0$. For $s=0$, factoring $1-\alpha^{m}$ gives

$$
1-\alpha^{m}=\left(1-\alpha^{m_{0}}\right)\left(\sum_{l=0}^{r-1} \alpha^{m_{0} l}\right) .
$$


Since $\alpha^{m_{0} l} \equiv 1 \bmod \mathfrak{p}$, for all $l \in\{0, \ldots, r-1\}$, we deduce

$$
v_{\mathfrak{p}}\left(1-\alpha^{m}\right)=v_{\mathfrak{p}}\left(1-\alpha^{m_{0}}\right)+v_{\mathfrak{p}}(r)=v_{0} .
$$

We now let $\beta=\alpha^{r m_{0}}$ and treat the case $s=1$. Writing $\beta=1+\lambda$, where $\lambda \in \mathfrak{p}$, we have that

$$
\frac{\beta^{p}-1}{\beta-1}=\frac{(1+\lambda)^{p}-1}{\lambda}=\sum_{k=1}^{p-1}\left(\begin{array}{l}
p \\
k
\end{array}\right) \lambda^{k-1}+\lambda^{p-1} .
$$

In the right-hand side, we have that $v_{\mathfrak{p}}(\lambda) \geq 1$, and $v_{\mathfrak{p}}\left(\lambda^{p-1}\right) \geq(p-1)>d \geq$ $v_{\mathfrak{p}}(p)$, so

$$
v_{\mathfrak{p}}\left(\sum_{k=1}^{p-1}\left(\begin{array}{l}
p \\
k
\end{array}\right) \lambda^{k-1}+\lambda^{p-1}\right)=v_{\mathfrak{p}}(p) .
$$

Hence, for $s=1$, we have

$$
v_{\mathfrak{p}}\left(1-\alpha^{m}\right)=v_{\mathfrak{p}}\left(1-\alpha^{m_{0} r}\right)+v_{\mathfrak{p}}\left(\frac{\beta^{p}-1}{\beta-1}\right)=v_{0}+v_{\mathfrak{p}}(p) .
$$

The statement now follows by induction on $s$, where the induction step from $s$ to $s+1$ is done as above (by replacing $\alpha$ by $\alpha^{p^{s}}$ ).

\section{Proof of Theorem 1.2}

Let $x=j(\tau), y=j\left(\tau^{\prime}\right)$ be two singular moduli of respective discriminants $\Delta$ and $\Delta^{\prime}$, with $\left\{\Delta, \Delta^{\prime}\right\}=\{-4 \cdot 23,-23\}$ or $\left\{\Delta, \Delta^{\prime}\right\}=\{-4 \cdot 31,-31\}$, such that

$$
A x^{m}+B y^{n}=C
$$

for some $A, B, C \in \mathbb{Q}^{\times}$and $m, n$ positive integers.

Both $x$ and $y$ are of degree 3 over $\mathbb{Q}$, and admit one real conjugate corresponding to the dominant $j$-value, and two complex conjugates. If $x$ is real, then $y$ is also real. Indeed, if not, then, together with (4.1), we have

$$
A x^{m}+B \bar{y}^{n}=C \text {. }
$$

We obtain that $y^{n}=\bar{y}^{n}$, which contradicts Lemma 2.1.

The equation (4.1) implies that $\mathbb{Q}\left(x^{m}\right)=\mathbb{Q}\left(y^{n}\right)$; hence, $\mathbb{Q}(x)=\mathbb{Q}(y)$ by Lemma 2.1. In particular, the Galois orbit of $(x, y)$ over $\mathbb{Q}$ has exactly 3 elements, and each conjugate of $x$ occurs exactly once as the first coordinate of a point in the orbit, just as each conjugate of $y$ occurs exactly once as the second coordinate.

We denote by $\left(x_{1}, y_{1}\right),\left(x_{2}, y_{2}\right),\left(x_{3}, y_{3}\right)$ the conjugates of $(x, y)$, with $x_{1}, y_{1}$ real, and $x_{2}, x_{3}$, respectively $y_{2}, y_{3}$, are complex conjugates. By (4.1) again, the points $\left(x_{i}^{m}, y_{i}^{n}\right), i \in\{1,2,3\}$, are collinear. We can write the relation of collinearity of these points in one of the following two ways:

$$
\begin{gathered}
\left|\begin{array}{lll}
1 & x_{1}^{m} & y_{1}^{n} \\
1 & x_{2}^{m} & y_{2}^{n} \\
1 & x_{3}^{m} & y_{3}^{n}
\end{array}\right|=0 \\
\left(\frac{x_{1}}{x_{2}}\right)^{-m}\left(\frac{y_{1}}{y_{2}}\right)^{n}=\frac{1-\left(\frac{y_{3}}{y_{2}}\right)^{n}-\left(\frac{x_{3}}{x_{1}}\right)^{m}}{1-\left(\frac{y_{3}}{y_{1}}\right)^{n}-\left(\frac{x_{3}}{x_{2}}\right)^{m}} .
\end{gathered}
$$


We focus first on the case $\left\{\Delta, \Delta^{\prime}\right\}=\{-4 \cdot 23,-23\}$, and we detail afterwards the slight differences in the treatment of the case $\left\{\Delta, \Delta^{\prime}\right\}=\{-4 \cdot 31,-31\}$. We denote by $L$ the Galois closure of $\mathbb{Q}(x)=\mathbb{Q}(y)$, which by definition contains all $x_{i}$ 's and $y_{i}$ 's.

As announced above, we consider the case $\Delta=4 \Delta^{\prime}=-4 \cdot 23$.

Using PARI, one can find a prime ideal $\mathfrak{p}$ of $\mathcal{O}_{L}$ over $p=23$ such that $\mathfrak{p} \mid x_{2} \mathcal{O}_{L}$, $\mathfrak{p} \mid x_{3} \mathcal{O}_{L}$, but $\mathfrak{p} \nmid x_{1} y_{2} y_{3} \mathcal{O}_{L}$. Hence, modulo $\mathfrak{p}^{m}$, the equation (4.2) becomes

$$
1-\alpha^{n}=0 \bmod \mathfrak{p}^{m},
$$

with $\alpha=y_{3} / y_{2}$. On the one hand, we deduce that $m \leq v_{\mathfrak{p}}\left(1-\alpha^{n}\right)$. On the other hand, we apply Proposition 3.2. checking first that $1-\alpha=0 \bmod \mathfrak{p}$, $v_{\mathfrak{p}}(1-\alpha)=1, v_{\mathfrak{p}}(p)=2<6<22=p-1$; writing $m=p^{s} r$ with $\operatorname{gcd}(p, r)=1$, we get

$$
v_{\mathfrak{p}}\left(1-\alpha^{m}\right)=s v_{\mathfrak{p}}(p)+1=2 s+1 .
$$

Consequently,

$$
m \leq 2 \frac{\log n}{\log 23}+1 .
$$

Next, we want to estimate the expression on the right-hand side of (4.3) in terms of $m$ and $n$ (in fact, only in terms of $n$ thanks to (4.4)), in order to obtain a bound on $n$. The principal difficulty is to find a lower bound of the absolute value of its denominator. Since $y_{3} / y_{1}$ is pretty close to 0 , it depends essentially on the quantity $1-\beta^{m}$ with $\beta=x_{3} / x_{2}$. Noticing that $|\beta|=1$ and $\beta$ is not a root of unity, then according to Theorem 3.1, there exists a constant $c_{1}(\beta)>0$ such that

$$
\left|1-\beta^{m}\right|>0.99 \mathrm{e}^{-c_{1}(\beta)(\log m)^{2}} .
$$

Explicitly, for $m \geq 13$, we can choose $c_{1}(\beta)=4973.14$. It follows that

$$
\begin{aligned}
\left|1-\left(\frac{y_{3}}{y_{1}}\right)^{n}-\left(\frac{x_{3}}{x_{2}}\right)^{m}\right| & >0.99 \mathrm{e}^{-4973.15(\log m)^{2}}-\left|\frac{y_{3}}{y_{1}}\right|^{n} \\
& >0.99 \mathrm{e}^{-4973.14\left(\log \left(2 \frac{\log n}{\log 23}+1\right)\right)^{2}}-\left|\frac{y_{3}}{y_{1}}\right|^{n}
\end{aligned}
$$

(recall the inequality (4.4)). By a quick calculation, we observe that the last term of the previous inequality is positive provided that $n>2074$. More specifically, if $n>2075$, then

$$
\left|1-\left(\frac{y_{3}}{y_{1}}\right)^{n}-\left(\frac{x_{3}}{x_{2}}\right)^{m}\right|>0.98 \mathrm{e}^{-4973 \cdot 14\left(\log \left(2 \frac{\log n}{\log 23}+1\right)\right)^{2}} .
$$

Finally, for $m \geq 13$ and $n>2075$, we have

$$
\left|\frac{x_{1}}{x_{2}}\right|^{-m}\left|\frac{y_{1}}{y_{2}}\right|^{n} \leq 2.05 \mathrm{e}^{4973.14\left(\log \left(2 \frac{\log n}{\log 23}+1\right)\right)^{2}},
$$

and

$$
\begin{aligned}
-\left(2 \frac{\log n}{\log 23}+1\right) \log \left|\frac{x_{1}}{x_{2}}\right|+n \log \left|\frac{y_{1}}{y_{2}}\right| \leq & \log 2.05 \\
& +4973.14\left(\log \left(2 \frac{\log n}{\log 23}+1\right)\right)^{2} .
\end{aligned}
$$


This last inequality yields $n \leq 2092$, and then (4.4) gives $m \leq 5$. This is in contradiction with the previous assumptions $m \geq 13$ and $n>2075$. Therefore, either $m<13$ or $n \leq 2075$. In both cases, $m<13$, and for each possible $m$, we can explicitly compute a constant $c_{2}(m)$ such that

$$
\left|\frac{x_{1}}{x_{2}}\right|^{-m}\left|\frac{y_{1}}{y_{2}}\right|^{n} \leq c_{2}(m)
$$

This allows to bound $n$. The table below summarizes all constants $c_{2}(m)$ and all bounds we obtain.

Table 4.1: Constants $c_{2}(m)$ and bounds on $n$ for each $m<13$, in the case $\Delta=4 \Delta^{\prime}=-4 \cdot 23$

\begin{tabular}{c|c|c}
$m$ & $c_{2}(m)$ & Upper bound of $n$ \\
\hline 1 & 1.15 & 2 \\
\hline 2 & 1.21 & 5 \\
\hline 3 & 11.97 & 8 \\
\hline 4 & 1.10 & 10 \\
\hline 5 & 1.28 & 13 \\
\hline 6 & 6.00 & 16 \\
\hline 7 & 1.07 & 18 \\
\hline 8 & 1.38 & 21 \\
\hline 9 & 4.02 & 24 \\
\hline 10 & 1.04 & 26 \\
\hline 11 & 1.50 & 29 \\
\hline 12 & 3.04 & 32
\end{tabular}

Again, inequality (4.4) eliminates all entries of Table 4.1 with $m \geq 3$. Consequently, either $m=1$ and $n \leq 2$, or $m=2$ and $n \leq 5$. For each of these remaining couples $(m, n)$, a direct calculation shows that the determinant in equation (4.2) does not vanish.

To finish, we repeat this process for the case $\Delta=4 \Delta^{\prime}=-4 \cdot 31$. In this case, one can find a prime ideal $\mathfrak{p}$ of $\mathcal{O}_{L}$ over $p=11$ such that $\mathfrak{p}\left|x_{2} \mathcal{O}_{L}, \mathfrak{p}\right| x_{3} \mathcal{O}_{L}$, but $\mathfrak{p} \nmid x_{1} y_{2} y_{3} \mathcal{O}_{L}$ as before, and we get

$$
m \leq \frac{\log n}{\log 11}+2 .
$$

We obtain as well, for $m \geq 13$ and $n>1440$,

$$
\left|\frac{x_{1}}{x_{2}}\right|^{-m}\left|\frac{y_{1}}{y_{2}}\right|^{n} \leq 2.05 \mathrm{e}^{4820.16\left(\log \left(\frac{\log n}{\log 11}+2\right)\right)^{2}},
$$

then

$-\left(\frac{\log n}{\log 11}+2\right) \log \left|\frac{x_{1}}{x_{2}}\right|+n \log \left|\frac{y_{1}}{y_{2}}\right| \leq \log 2.05+4820.16\left(\log \left(\frac{\log n}{\log 11}+2\right)\right)^{2}$,

which yields $n \leq 1720$ and $m \leq 5$; again a contradiction. For each possible $m<13$, we compute a constant $c_{2}(m)$ as defined above, and we deduce a bound on $n$. Here is the table: 
Table 4.2: Constants $c_{2}(m)$ and bounds on $n$ for each $m<13$, in the case $\Delta=4 \Delta^{\prime}=-4 \cdot 31$

\begin{tabular}{c|c|c}
$m$ & $c_{2}(m)$ & Upper bound of $n$ \\
\hline 1 & 1.13 & 3 \\
\hline 2 & 1.25 & 6 \\
\hline 3 & 6.17 & 10 \\
\hline 4 & 1.06 & 13 \\
\hline 5 & 1.44 & 16 \\
\hline 6 & 3.13 & 19 \\
\hline 7 & 1.02 & 22 \\
\hline 8 & 1.76 & 26 \\
\hline 9 & 2.13 & 29 \\
\hline 10 & 1.01 & 32 \\
\hline 11 & 2.33 & 36 \\
\hline 12 & 1.65 & 39
\end{tabular}

Inequality (4.5) eliminates all entries of Table 4.2 with $m \geq 3$. Consequently, either $m=1$ and $n \leq 3$, or $m=2$ and $n \leq 6$. Each of these remaining possibilities can be excluded by a direct calculation showing that the respective determinant does not vanish.

\section{References}

[1] B. Allombert, Yu. Bilu, A. Pizarro-Madariaga, CM-Points on Straight Lines, in: C. Pomerance, M. T. Rassias (editors), Analytic Number Theory In Honor of Helmut Maier's 60th Birthday, 1-18, Springer, 2015.

[2] Y. André, Finitudes des couples d'invariants modulaires singuliers sur une courbe algébrique plane non modulaire, J. Reine Angew. Math. 505 (1998), 203-208.

[3] Yu. Bilu, G. Hanrot, P. M. Voutier, M. Mignotte, Existence of primitive divisors of Lucas and Lehmer numbers, J. reine angew. Math. 539 (2001), 75-122.

[4] Yu. Bilu, D. Masser, U. Zannier, An effective "Theorem of André" for CM-points on a plane curve, Math. Proc. Cambridge Philos. Soc. 154 (2013), 145-152.

[5] Yu. Bilu, F. Luca, A. Pizarro-Madariaga, Rational Products of Singular Moduli, Journal of Number Theory 158 (2016), 397-410.

[6] E. Bombieri, W. Gubler, Heights in Diophantine Geometry, Cambridge University Press, 2006.

[7] D. A. Cox, Primes of the form $x^{2}+n y^{2}$, Wiley, NY, 1989.

[8] L. Kühne, An effective result of André-Oort type II, Acta Arith. 161 (2013), 1-19.

[9] M. Laurent, M. Mignotte, Y. Nesterenko, Formes linéaires en deux logarithmes et déterminants d'interpolation, J. Number Th. 55 (1995), 285-321.

[10] A. Riffaut, Equations with powers of singular moduli, arXiv:1710.03547, 2017.

[11] The PARI Group, PARI/GP version 2.7.1 (2014), Bordeaux; available from http://pari.math.u-bordeaux.fr/ 